\title{
GNSS SATELLITE VISIBILITY ANALYSIS BASED ON 3D SPATIAL INFORMATION IN URBAN AREAS
}

\author{
Ya-Hsuan Lu ${ }^{1}$, Jen-Yu Han ${ }^{2}$ \\ ${ }^{1}$ Dept. of Civil Engineering, National Taiwan University, No.1, Sec. 4, Roosevelt Rd., Da an Dist., Taipei City 10617, Taiwan, \\ -r07521802@ntu.edu.tw \\ ${ }^{2}$ Dept. of Civil Engineering, National Taiwan University, No.1, Sec. 4, Roosevelt Rd., Da an Dist., Taipei City 10617, Taiwan,
} - jyhan@ntu.edu.tw

KEY WORDS: Satellite Visibility Analysis, Satellite Positioning Evaluation, Topographic Effect, Terrain Data Processing, DOP

\begin{abstract}
:
Global Navigation Satellite System (GNSS) is a matured modern technique for spatial data acquisition. Its performance has a great correlation with GNSS receiver position. However, high-density building in urban areas causes signal obstructions and thus hinders GNSS's serviceability. Consequently, GNSS positioning is weakened in urban areas, so deriving proper improvement resolutions is a necessity. Because topographic effects are considered the main factor that directly block signal transmission between satellites and receivers, this study integrated aerial borne LiDAR point clouds and a 2D building boundary map to provide reliable 3D spatial information to analyze topographic effects. Using such vector data not only reflected high-quality GNSS satellite visibility calculations, but also significantly reduced data amount and processing time. A signal obstruction analysis technique and optimized computational algorithm were also introduced. In conclusion, this paper proposes using superimposed column method to analyze GNSS receivers' surrounding environments and thus improve GNSS satellite visibility predictions in an efficient and reliable manner.
\end{abstract}

\section{INTRODUCTION}

\subsection{Satellite Visibility Analysis}

Global Navigation Satellite System (GNSS) has been widely used in the surveying field, as well as in other engineering applications. Therefore, knowing how to increase positioning quality is crucial. One of the main positioning factors is satellite and receiver connection, called satellite visibility, which depends on the line between the receiver on the ground and the satellite in the sky.

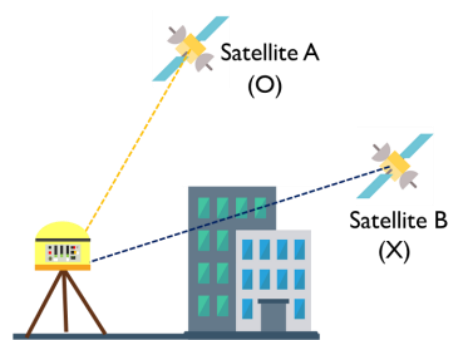

Figure 1. Illustration of signal obstruction from satellite to receiver

As in Figure 1, the line between the receiver and Satellite A is clear, which means that Satellite A is visible to the ground station. However, the line between the receiver and Satellite B is blocked by a building, meaning the signal transmission is disturbed. There are many other types of obstacles, such as urban canyons, mountainous areas, or areas of dense vegetation, all of which lead to a similar lack of satellite coverage (Ackermann et al., 2013). To distinguish whether a satellite is visible or not, though, we must first analyze the station's location to derive the maximum visual elevation angle in all directions.

Many scholars have already begun to explore satellite visibility analysis. For example, Verbree et al. (2004) combined cadastral maps with LiDAR to classify objects on the surface and reconstruct a building model. Next, they formed a point cloud of building roofs to calculate elevation angle. Verbree et al.'s (2004) results then used colored dots to indicate the percentage of satellite visibility, where Blue-Red represented $<60-100 \%$ availability (Figure 2).

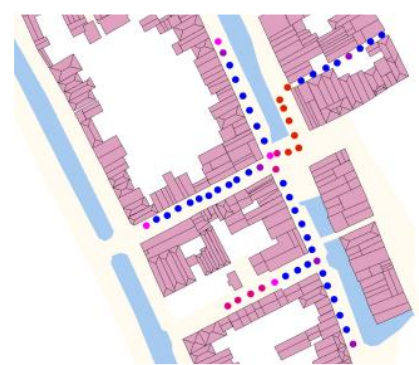

Figure 2. Satellite visibility percentage in Delft (Verbree et. al., 2004)

Taylor et al. (2007), meanwhile, established a DSM model that merged aerial photography and LiDAR into a realistic digital surface model to calculate the Line of Sight (LOS) vector. As shown in Figure 3, the green lines represent visibility and the red lines invisibility. Using LOS can thus determine the status between satellites and stations and aid decisions on whether to perform GPS surveying.

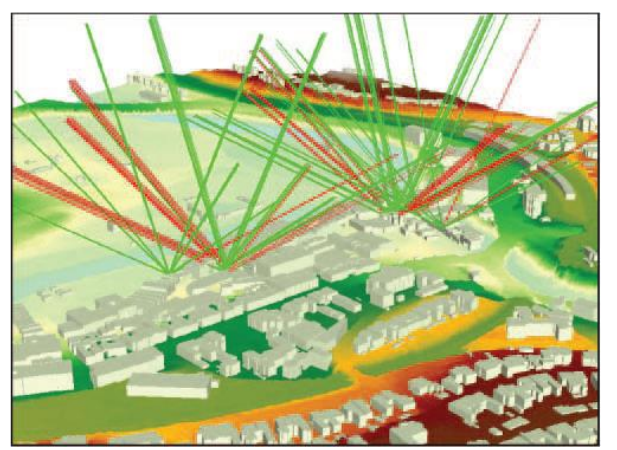

Figure 3. LOS of GPS satellite visibility (Taylor et al., 2007)

\subsection{Terrain Data Analysis}

To derive the elevation angle in each direction, the terrain data must first be processed. The two most commonly used data in terrain analysis are raster data and vector data. 


\section{(i) Raster Data}

Raster data uses grids or cells to store geometric information, such as aerial photographs, satellite images, and DSM. A higher cell resolution requires more space to store the data, affecting calculation efficiency. To improve this problem, Han and $\mathrm{Li}$ (2010) proposed the non-equal interval sampling method: if the terrain is more complicated, the satellite orbit's sampling time shorter, or the sampling point closer to the receiver, the sampling interval must be shorter. This method's advantage is that it can be more effective than equal interval sampling and reduce data calculation time.

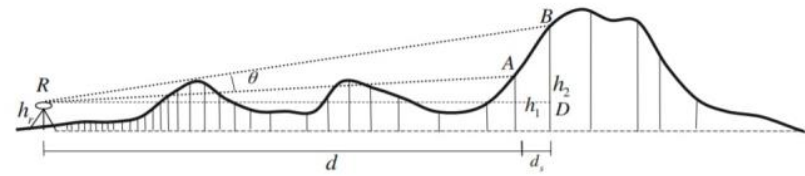

Figure 4. Non-equal interval sampling method (Han \& Li, 2010)

(ii) Vector Data

Vector data uses points, lines, and polygons to store geometric information. This type of data requires less space because only coordinates are stored. LiDAR is one of the main remote sensing technologies nowadays and can calculate the distance between the sensor and its target by launching a laser wave and measuring the time difference of the reflected wave. Another advantage of LiDAR is that it has high penetration, fast speed, and is unaffected by day or night hours.

LiDAR has already been widely used in 3D terrain models. For example, Rottensteiner and Briese (2002) used high-resolution LiDAR data to test the establishment of a small area DSM in Vienna (Figure 5).

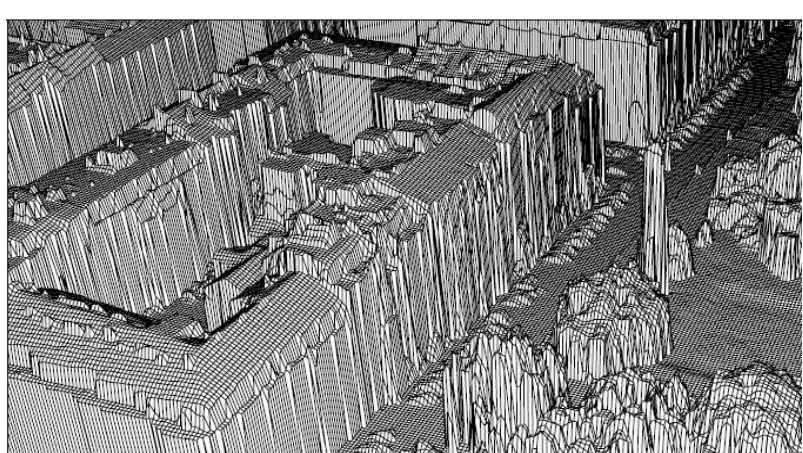

Figure 5. Using LiDAR to reconstruct a terrain model in Vienna (Rottensteiner \& Briese, 2002)

Traditionally, aerial photography is a rapid and low-cost way to acquire DSM in flat areas with small scales. Yet, due to the high building density in urban areas, the situation in cities is more complicated. Buildings make it very difficult to completely and clearly identify satellites. Furthermore, images can have problems such as image resolution, shadow area, depth discontinuity, material difference, etc. and sometimes even need manually selected object pairs (Zhou et al., 2004). This process thus becomes time- and cost-consuming. Rottensteiner and Briese (2002) suggested that LiDAR is the much more suitable choice in city areas because of the high density of point clouds. Buildings can be detected first in the point cloud, and then the edges of those buildings can be reconstructed to build a 3D model. Therefore, LiDAR is a more suitable resource for analyzing 3D models of complicated topographies.

\subsection{GNSS Positioning Quality Index}

The GNSS dilution of precision (DOP) is related to satellites' geometric spatial distribution and can be estimated from prior knowledge of satellite visibility along a planned route (Ackermann et al., 2013; Leick, 2004). DOP factors are functions of the diagonal elements from the posterior variance covariance matrix of the least square solution of the GNSS positioning, as shown in Eq. (1).

$$
\mathrm{Q}_{\mathrm{xx}}=\left[\begin{array}{cccc}
\sigma \mathrm{X}^{2} & \sigma \mathrm{XY} & \sigma \mathrm{XZ} & \sigma \mathrm{Xdt} \\
& \sigma \mathrm{Y}^{2} & \sigma \mathrm{YZ} & \sigma \mathrm{Ydt} \\
& & \sigma \mathrm{Z}^{2} & \sigma \mathrm{Zdt} \\
\text { sym. } & & & \sigma \mathrm{dt}^{2}
\end{array}\right]
$$

Table 1 shows five different DOP expressions: the vertical dilution of precision (VDOP) for height, the horizontal dilution of precision (HDOP) for horizontal positions, the time dilution of precision (TDOP) for time error accuracy between the receiver and the satellite, the positional dilution of precision (PDOP) for $3 \mathrm{D}$ coordinate accuracy, and the geometric dilution of precision (GDOP) for 3D coordinate and time accuracy.

Table 1. DOP Expressions (Leick, 2004)

$\mathrm{VDOP}=\sqrt{\sigma \mathrm{Z}^{2}}$
$\mathrm{HDOP}=\sqrt{\sigma \mathrm{X}^{2}+\sigma \mathrm{Y}^{2}}$
$\mathrm{PDOP}=\sqrt{\sigma \mathrm{X}^{2}+\sigma \mathrm{Y}^{2}+\sigma \mathrm{Z}^{2}}$
$\mathrm{TDOP}=\sqrt{\sigma \mathrm{dt}^{2}}$
$\mathrm{GDOP}=\sqrt{\sigma \mathrm{X}^{2}+\sigma \mathrm{Y}^{2}+\sigma \mathrm{Z}^{2}+\mathrm{c}^{2} * \sigma \mathrm{dt}^{2}}$

Calculating DOP in advance can give approximations of receiver location. It can also find the best satellite subsets on a given day.

\section{METHODOLGY}

\subsection{Vector Terrain Data Processing}

In this study, LiDAR data was used to analyze terrain. However, the raw LiDAR point cloud data was too large and contained many unnecessary parts. Therefore, preliminary data extraction was required, so we extracted the LiDAR points based on 2D boundaries and homothetic boundaries transformation.

\subsubsection{LiDAR Points Extraction Based on 2D Boundaries}

In urban areas, the obstacles caused by high building density that affect the connection between a satellite and its receiver are complex, so only building parts are required. Hence, using open data provided by the government, the LiDAR points and 2D building boundary map were combined and then the building sections extracted. As a result, the LiDAR building parts remained and the rest of the points removed. This served as an initial data reduction in keeping just the useful points.

\subsubsection{Homothetic Boundaries Transformation}

However, the extracted points may still have contained some useless points. For example, the points near the building boundary may have had some noise, such as reflection off a wall. To clear the noise points, the building boundary was rendered smaller. Modifying the building boundary into a smaller size ensured that the rest of the points were all in the rooftop area, which was important because the rooftop area can be used to calculate building height. 


\subsection{Superimposed Column Method}

Because urban areas have lots of buildings, the data amount is large. To effectively reduce this amount, the points that were already extracted were processed via a superimposed column method and stored in a vector database. As seen in Figure 6 and Figure 7, the superimposed column concept involves turning buildings into stacked columns and then storing the height information and coordinates of each. The greatest benefit of this method is that it can turn even 1 million LiDAR points into small data pieces, helping simplify calculations and storage. In addition, the superimposed column method kept the basic shape and height information of building rooftops.

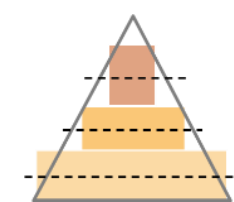

Superimposed column method (side view)
Figure 6

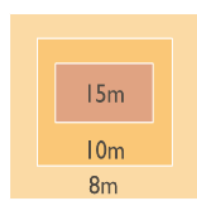

Figure 7.

Superimposed column method (top view)

\subsection{Maximum Elevation Angle Calculation}

After gathering the buildings' height and coordinate information, the final step was to derive the maximum elevation angle and find each direction's mask angle, as shown in Figure 8 and Figure 9.

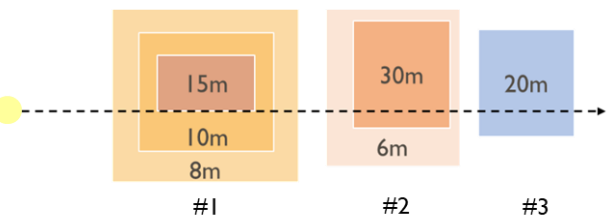

Figure 8. Maximum mask angle calculation (top view)

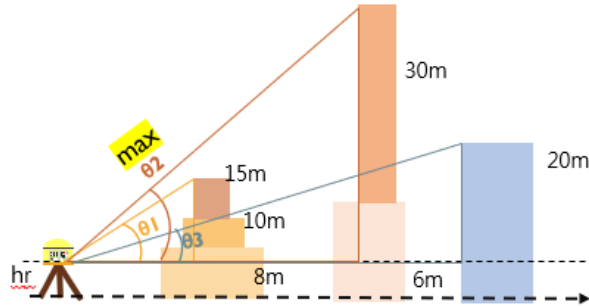

\#2

\#3

Figure 9. Maximum mask angle calculation (side view)

Elevation angle was derived from Eq.(2):

$$
\mathrm{El}=\arctan \left(\frac{\mathrm{H}}{\mathrm{D}}\right)
$$

where $E l$ is the elevation angle from the receiver to the building, $H$ is the building height - the receiver height, and $D$ is the distance between the receiver and the building.

Using Eq. (2), we learned the maximum elevation angle in each direction and treated them as mask angles. After finding all mask angles, we considered satellite position to draw the skyplot and estimate the DOP values. While completing all the above methods, the results had to be verified; calculation efficiency is also one of the evaluated performances.

\section{RESULTS AND DISCUSSION}

\subsection{Experiment Data}

The experiment data was twofold: a LiDAR point cloud (Figure 10) and a 2D building boundary map of National Taiwan University (NTU; Figure 11). The original LiDAR data contained $5,798,616$ points, and the building boundary map had 829 polygons.

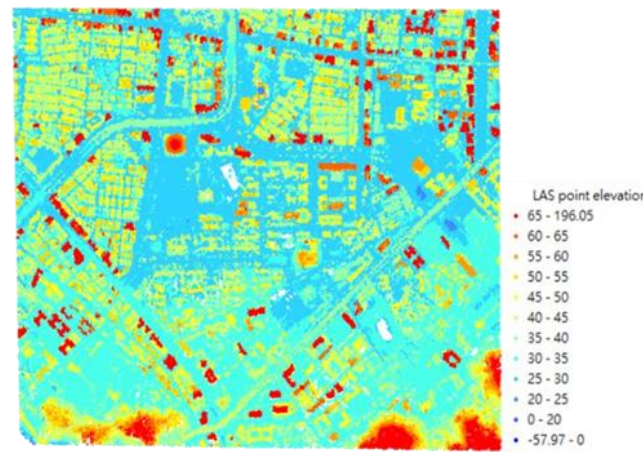

Figure 10. LiDAR point cloud of NTU

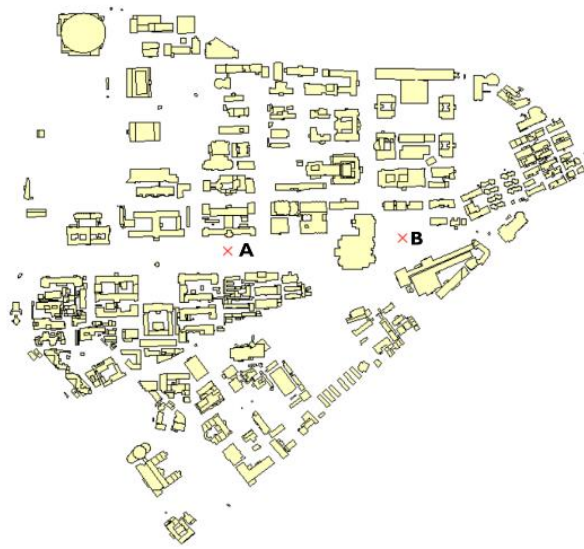

Figure 11.2D building boundary of NTU

Since the goal of this research was to find a suitable survey time for the GNSS receiver, the experiment specified two spaces as the receiver's location. In Figure 11, point A is surrounding by many buildings, so its visibility to the sky is seriously blocked. As for point $\mathrm{B}$, it is located in the school square, which is a much more open area than point A. For those two points, point A was a high masking point and point $\mathrm{B}$ was a low masking point.

\subsection{LiDAR Point Processing}

For the experiment, we only needed to analyze the obstacles in the city. Therefore, the first step was to take out the unnecessary building points. After using the 2D building boundary to remove the extraneous points inside it, the remaining points generated Figure 12. This lowered the amount of points from 5,798,616 to 430,896 . 


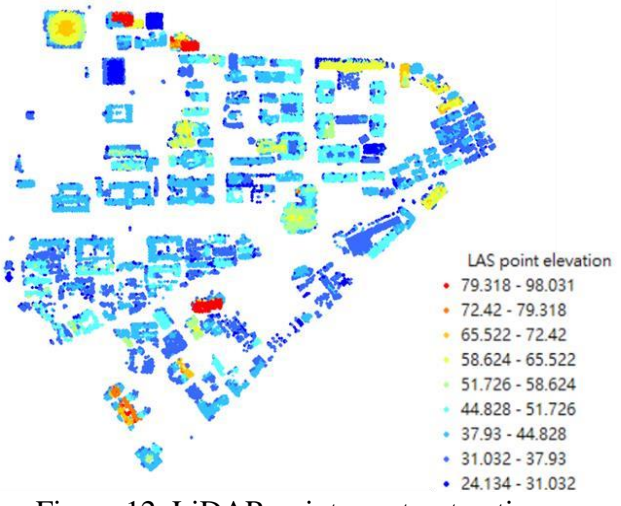

Figure 12. LiDAR points post-extraction

The second step required using homothetic transformation to create a new building boundary. Because the research only focused on the rooftop building area, the rest of the buildings were excluded. This process also ensured the removal of wall reflection points. Figure 13 indicates an example of the building boundary's homothetic transformation.

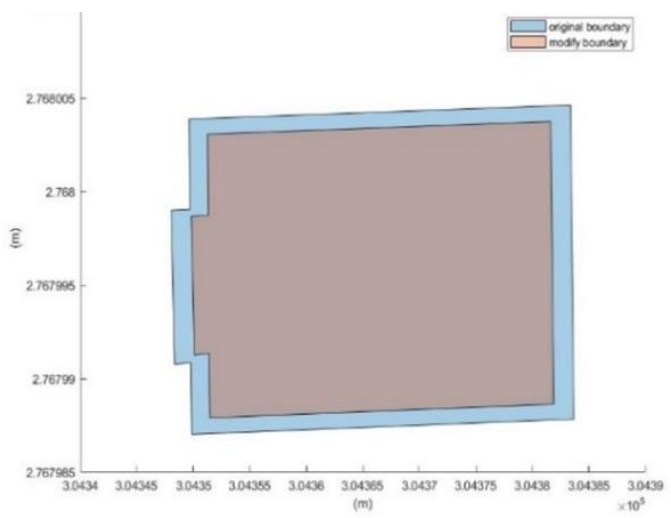

Figure 13. Example of building boundary homothetic transformation

The modified polygon is 0.9 times the original polygon due to Taiwan's general building wall width. Thereafter, only the points inside the modified polygon were kept.

\subsection{Building Superimposed Columns}

After the preliminary arrangement of the point cloud, the remaining points were treated as building roofs. Although it was processed, the amount of data was still large, so this study used superimposed columns to simplify the data and save it into a vector database, which is easier to process.

The meaning of the superimposed columns is to set a quadrilateral with four corner points as a layer, turning one building into pieces of stacked layers like in Figure 6. A quadrilateral can frame all points in the same layer, so the four corner points were stored as $\mathrm{x}-\mathrm{y}$ coordinates; the layer height values were also recorded.

For example, in Figure 14, the red dots represent the LiDAR data, containing 528 points. The first layer starts from the bottom of the points. There is a 0.5 meter range between each layer. As a result, it places all $529 \mathrm{LiDAR}$ points into 3 layers, which means only 3 (layer) x 4 (corner points) $=12$ points need to be stored.

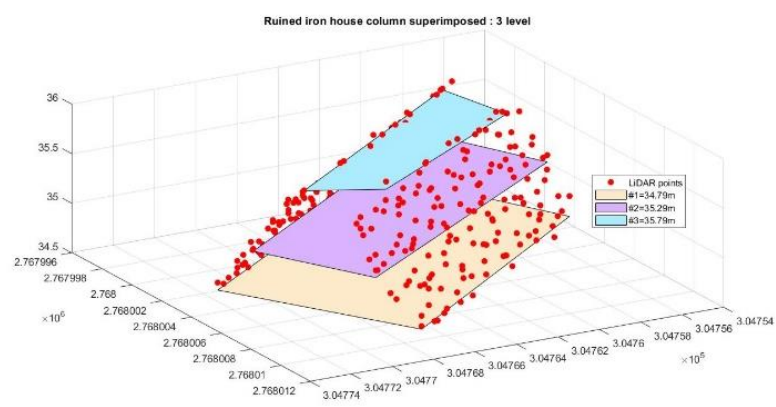

Figure 14. Superimposed building column sample

Table 2 shows the amount of data for experimental points A and $\mathrm{B}$ after the columns were superimposed.

Table 2. Data amount after column superimposition

\begin{tabular}{cc}
\hline Point A & 76,785 (layers) \\
Point B & 71,967 (layers) \\
\hline
\end{tabular}

\subsection{Mask Angle Calculation}

The final step was to compute the maximum elevation angle of each direction (Figure 15). We set a buffer at a radius of 1,000 meters. Each 1 degree was calculated its own mask angle. Moreover, it only calculated the building higher than the last one, which means that not all buildings were calculated with the elevation angle. With this conditional expression, the calculation time was expedited.

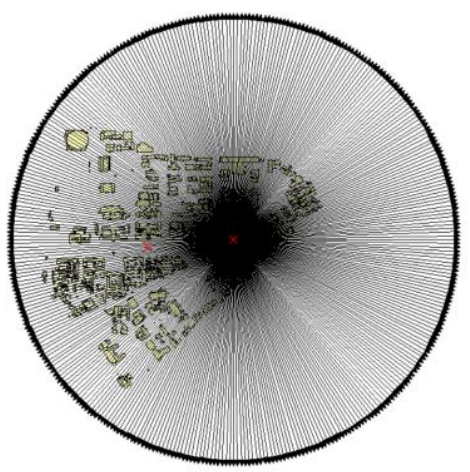

Figure 15. Calculation of elevation angle from each direction

With the maximum elevation angle for each direction calculated, we drew the skyplot for the receiver points. Point A's skyplot is shown in Figure 16 and point B's in Figure 17. The center of each circle indicates the receiver's position.

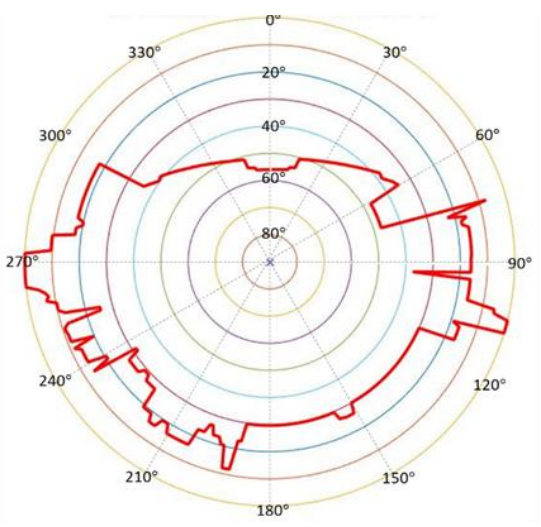

Figure 16. Skyplot of point A 


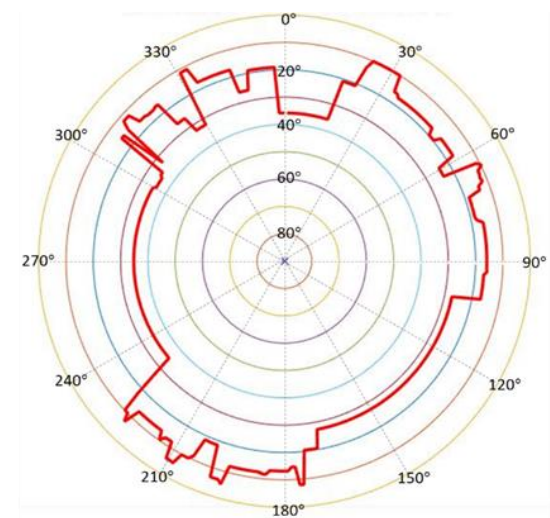

Figure 17. Skyplot of point B

\subsection{Comparing the Results}

To verify the experiment's results, the research tested two additional datasets: the original LiDAR data and raster data made from the original LiDAR data (Figure 18). The raster resolution was $1 \mathrm{mx} 1 \mathrm{~m}$.

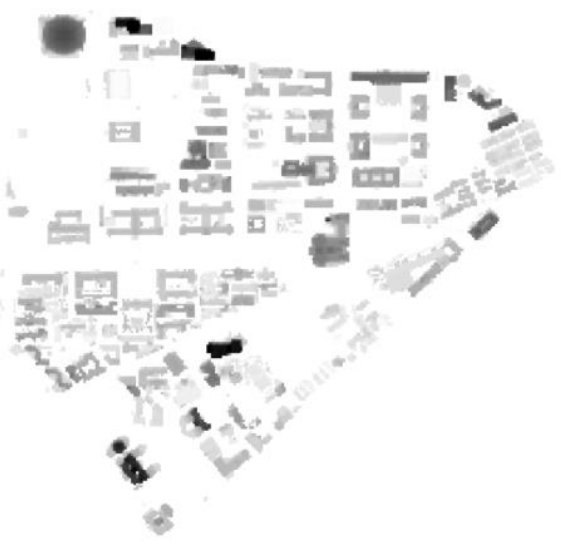

Figure 18. Raster data made from the original LiDAR data

It also used the three different datasets to calculate the mask angle in each direction to compare results and time efficiency.

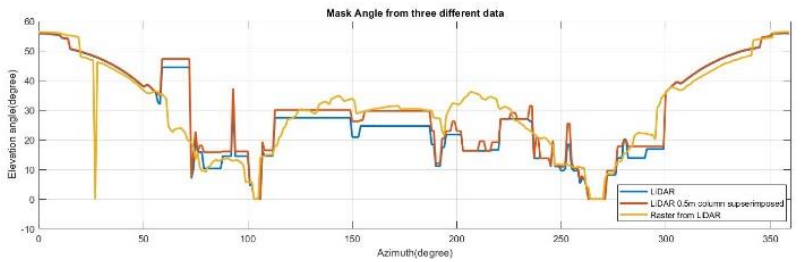

Figure 19. Point A's mask angle from three different dataset

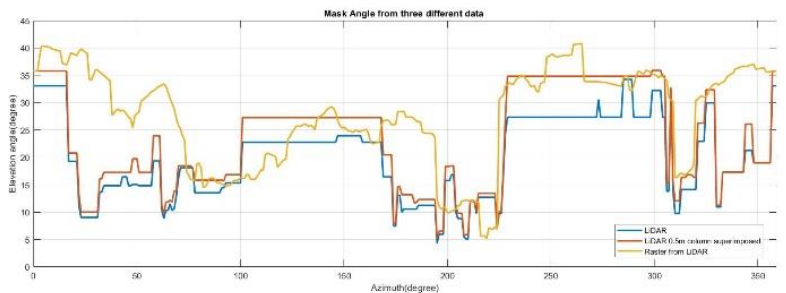

Figure 20. Point B's mask angle from three different datasets

In Figure 19 and Figure 20, the blue line indicates the original LiDAR data, the orange line the superimposed column method, and the yellow line the raster data. The $\mathrm{x}$-axis represents the azimuth and the $y$-axis each direction's elevation angle. It is clear that the blue and orange lines have a similar trend. It is also worth noting that the orange line is typically higher than the blue line, which might be because the original LiDAR data used average height to represent building height.

However, the raster data results are quite different from the others. The reason may be that when the grid was produced, the point cloud was projected onto a blank grid, and the average height of all points in the range were used as the grid values. Hence, the average height may have caused some errors in certain grids.
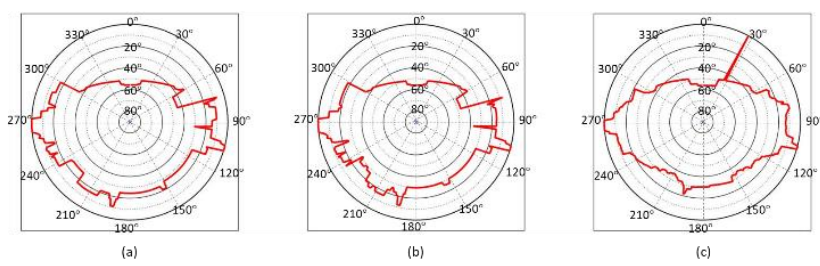

Figure 21. Skyplot of point A per the three different datasets
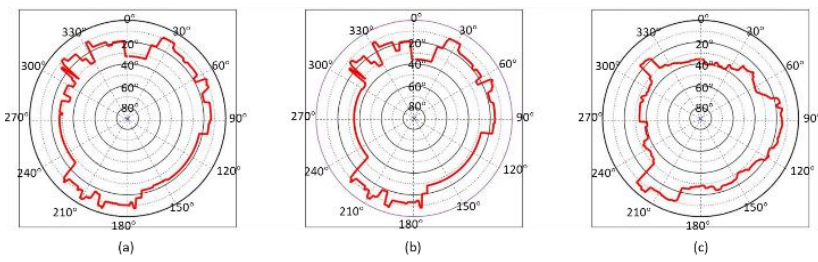

Figure 22. Skyplot of point B per the three different datasets

Figure 21 and Figure 22 show point A and point B's skyplots per the three different datasets, where (a) is the original LiDAR data, (b) is the LiDAR data with superimposed columns, and (c) is the raster data. Once again, the vector data featured more identical results.

Another way we checked the three datasets was with RMSD. RMSD is used to measure the differences between predicted and observed values. In this study's context, the predicted value should be the original LiDAR data. Table 3 shows the RMSD of each method. Just as with the trends in Figure 19 and Figure 20, the vector data had a very similar RMSD, while the raster data was different.

Table 3. RMSD comparison

\begin{tabular}{lcc}
\hline & LiDAR & \& \\
& LiDAR with & LiDAR \\
& $\begin{array}{c}\text { Superimposed } \\
\text { Columns }\end{array}$ & Raster LiDAR \\
& $2.583^{\circ}$ & \\
\hline RMSD_A & $4.099^{\circ}$ & $7.800^{\circ}$ \\
RMSD_B & & $11.182^{\circ}$ \\
\hline
\end{tabular}

Tables 4 and Table 5 list the amount of data and calculation time that correspond to each method. For point A, after column superimposition, the data was $3.46 \%$ of the amount of LiDAR data, and the time required was only $0.003 \%$ of the original. For point $B$, the data after column superimposition was $2.53 \%$ of the original LiDAR data points, and time efficiency was $0.003 \%$ of the original. Although the original LiDAR points and the LiDAR points after column superimposition were all vector data, the data amount still made a tremendous difference. On the other hand, no matter at which test point, the raster data took much more time to calculate than the original LiDAR data. The data storage was also different, with the raster data taking $5.05 \mathrm{~GB}$ but the LiDAR with superimposed columns data only $800 \mathrm{~KB}$. The raster data also needed much more space and time to calculate than the LiDAR data with superimposed columns.

Table 4. Data information of point A 


\begin{tabular}{cccc}
\hline Data Type & $\begin{array}{c}\text { Original } \\
\text { LiDAR }\end{array}$ & $\begin{array}{c}\text { LiDAR with } \\
\mathbf{0 . 5} \mathbf{~ m} \\
\text { Superimposed } \\
\text { Columns }\end{array}$ & $\begin{array}{c}\text { Raster } \\
(\mathbf{1 ~ m ~ x ~ 1 ~} \\
\mathbf{~ m})\end{array}$ \\
\hline \multirow{2}{*}{ Data amount } & $\begin{array}{c}2,216,504 \\
\text { (pts) }\end{array}$ & $\begin{array}{c}76,785 \\
\text { (layers) }\end{array}$ & $\begin{array}{c}1235 \times \\
(\text { pixel })\end{array}$ \\
Time & $2314.443(\mathrm{~s})$ & $0.067(\mathrm{~s})$ & $39.814(\mathrm{~s})$ \\
\hline
\end{tabular}

Table 5. Data information of point B

\begin{tabular}{cccc}
\hline $\begin{array}{c}\text { Data } \\
\text { Type }\end{array}$ & $\begin{array}{c}\text { Original } \\
\text { LiDAR }\end{array}$ & $\begin{array}{c}\text { LiDAR with 0.5 m } \\
\text { Superimposed Columns }\end{array}$ & $\begin{array}{c}\text { Raster } \\
(\mathbf{1 ~ m ~ x ~} \\
\mathbf{1 ~ m})\end{array}$ \\
\hline Data & $2,839,944$ & 71,967 & $1235 \times$ \\
amount & $(\mathrm{pts})$ & (layers) & $\begin{array}{c}1155 \\
(\mathrm{pixel})\end{array}$ \\
Time & $\begin{array}{c}2047.494 \\
(\mathrm{~s})\end{array}$ & $0.064(\mathrm{~s})$ & $\begin{array}{c}39.408 \\
(\mathrm{~s})\end{array}$ \\
\hline
\end{tabular}

Finally, mask angle proved important because it can reflect the real topographic situation around the receiver. The reason why the study analyzed terrain data was because mask angle might be underestimated. Figure 23 is a skyplot of point A from 2020/3/25 00:00 (UTC). When the mask angle was $15^{\circ}$, the number of visible satellites was 8

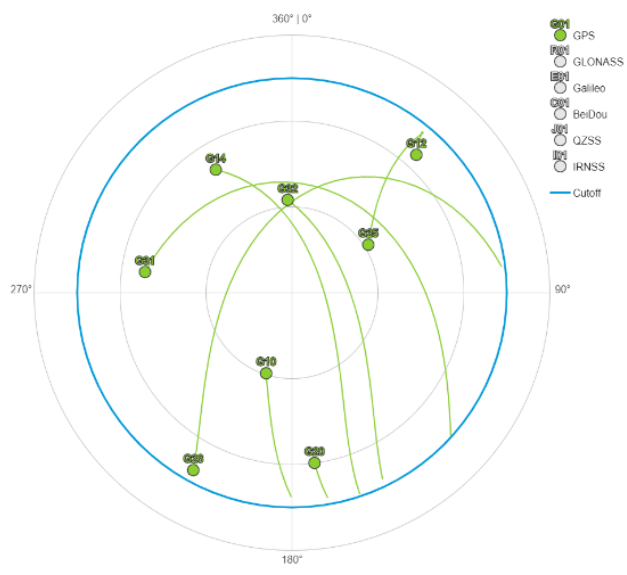

Figure 23. Skyplot from Trimble GNSS planning online

Yet, after analyzing the true 3D terrain data, this experiment showed the true mask angle should resemble that in Figure 16. Hence, after combining satellite position and mask angle, the corrected skyplot was generated, with the number of visible satellites 3 instead of 8 (Figure 24).

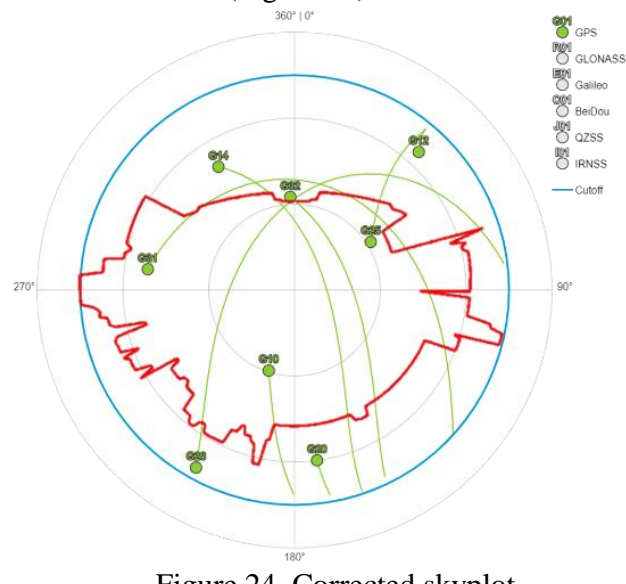

Figure 24. Corrected skyplot

\section{CONCLUSIONS AND FUTURE WORK}

The aim of this research was to use the LiDAR superimposed column method to find the best satellite observation time of day. The current results indicate that such vector data uses less store space and has a much faster processing speed compared to the same calculation performed with raster data. Furthermore, compare to the original LiDAR data and the raster data, the superimposed column method proposed by the research is the one with the smallest amount of data and the fastest speed. When the buildings are regenerated as columns, the data amount is roughly $3 \%$ of the original and takes only $0.003 \%$ of the original data's calculation time. In addition, the RMSD between the LiDAR superimposed column data and original LiDAR data is about $3.3^{\circ}$, showing the LiDAR superimposed column method is a quick and effective method to predict satellite visibility.

In the future, researchers should continue to improve the superimposed column method's accuracy. Additionally, the research may benefit from actual field GNSS surveying based on experimental results; the DOP should be estimated in advance and then compared with the actual DOP obtained during the GNSS field survey, ensuring that the research is supported by actual evidence.

\section{REFERENCES}

Ackermann, S., Angrisano, A., Del Pizzo, S., Gaglione, S., Gioia, C., \& Troisi, S.,2013. Digital surface models for GNSS mission planning in critical environments. Journal of Surveying Engineering, 140(2):04014001.

Guoqing Zhou, C. Songa, J. Simmersb, P. Cheng, 2004. Urban 3D GIS from LiDAR and digital aerial images. Computers \& Geosciences 30.4, p.345-353.

Han, J. Y., \& Li, P. H., 2010. Utilizing 3-D topographical information for the quality assessment of a satellite surveying. Applied Geomatics, 2(1):21-32.

Leick, A., 2004. GPS satellite surveying third edition, John Wiley \& Sons., Hoboken, New Jersey, p.252

Rottensteiner, F., \& Briese, C., 2002. A new method for building extraction in urban areas from highresolution LIDAR data. International Archives of Photogrammetry Remote Sensing and Spatial Information Sciences, 34(3/A):295-301.

Taylor, G., Li, J., Kidner, D., Brunsdon, C., \& Ware, M. , 2007. Modelling and prediction of GPS availability with digital photogrammetry and LiDAR. International Journal of Geographical Information Science, 21(1):1-20.

Verbree, E., Tiberius, C. and Vosselman, G., 2004. Combined GPS-Galileo positioning for location based services in urban environment. In Proceedings of the Location Based Services \& Telecartography Symposium 2004 (Austria: Vienna University of Technology), p.99-107. 